\title{
Diagnostic Approach for Suspected Allergic Cutaneous Reaction to a Permanent Tattoo
}

\author{
Silvestre JF, González-Villanueva I \\ Servicio de Dermatología, Hospital General Universitario de Alicante, Alicante, Spain
}

J Investig Allergol Clin Immunol 2019; Vol. 29(6): 405-413

doi: 10.18176/jiaci.0383

\begin{abstract}
The last few decades have seen a notable increase in the number of people who have a tattoo. This practice is not free from complications. Most adverse effects appear early and are temporary, although they can occasionally develop later and be permanent and serious. Recent research has generated new knowledge on the composition of inks, their degradation over time, the immune activity that is stimulated, and the various clinical disorders that can arise. This information enables better approaches to diagnosis and management when complications arise. Diagnosing allergic reactions to permanent tattoo ink can be very challenging. This review aims to identify clinical and histological clues to help practitioners differentiate allergic reactions from other complications. We discuss the yield and appropriateness of skin tests and biopsies and propose an algorithm to guide the diagnostic process.
\end{abstract}

Key words: Tattoo. Adverse effect. Skin. Allergy. Patch testing. Biopsy. Diagnosis.

\section{Resumen}

En las últimas décadas ha aumentado ostensiblemente el número de personas que se realiza un tatuaje en la piel. Esta técnica no está exenta de complicaciones. La mayoría son precoces y transitorias, pero, en ocasiones, son tardías, permanentes y graves. En los últimos años, los avances en el conocimiento de la composición de tintas, de los procesos de degradación de las mismas tras el tatuado, de la actividad inmunitaria que se desencadena y la descripción de una clasificación en patrones clínicos, nos han permitido realizar un mejor enfoque diagnóstico y manejo terapéutico de estas complicaciones.

El diagnóstico de las reacciones alérgicas a las tintas de los tatuajes permanentes supone un verdadero reto. En esta revisión intentaremos dar claves clínicas e histológicas que nos ayudan a diferenciar las reacciones alérgicas del resto de complicaciones. Discutiremos la rentabilidad e idoneidad de las pruebas epicutáneas y de la biopsia cutánea, y propondremos un algoritmo diagnóstico.

Palabras clave: Tatuaje. Complicación. Piel. Alergia. Pruebas epicutáneas. Biopsia. Diagnóstico. 


\section{Introduction}

Tattooing is a decorative skin technique that has been used with various objectives for millennia. Tattoos are common in a number of cultures, and the practice has risen in popularity since the 1970 s, especially in young people [1]. By some estimates, $10 \%$ to $20 \%$ of people in Western countries now have at least 1 tattoo [2]. The deposition of pigment particles and other components of ink in the dermis induces a response. Most reactions are due either to an inflammatory defense response to foreign substances or local toxicity following exposure to irritants. In clinical practice, this translates into the appearance of mild symptoms within a month of receiving the tattoo (pruritus, swelling, or hair loss) in about 1 in every 5 individuals [3]. Most of these complaints do not require a visit to a doctor, as tattooists are familiar with and know how to manage them. Other reactions are serious, persistent, and chronic and tend to appear later and more rarely. Some studies have estimated that $6 \%$ to $8 \%$ of people who have a tattoo are affected by this type of complication $[4,5]$ and seek specialist care. However, the right diagnostic approach is difficult, as it must take into account multiple clinical entities ranging from infections, tumors, allergic processes, and foreign body reactions to manifestations of systemic diseases or isomorphic phenomena arising from other skin conditions.

This review aims to provide clinical and histological clues that can help clinicians to differentiate between allergic reactions to tattoos and other complications. We also discuss the yield and appropriateness of skin tests and biopsies and propose an algorithm to guide the diagnostic process based on our clinical experience [6].

\section{Clinical Manifestations}

A detailed history is essential for reaching a diagnosis and should include the following: an investigation of signs and symptoms related to lesions in the tattooed area; questions on the patient's personal medical history, with an emphasis on previous dermatoses; and a comprehensive account of the conditions in which the patient received the tattoo (Table 1) [7].

An important detail that can orient the diagnosis is the time between getting the tattoo and onset of symptoms [6]. This can help clinicians to classify the reaction as early (within a month of being tattooed) and late (appearing months or even years afterward) [8]. The most common early complications are inflammatory reactions, which occur secondary to cutaneous exposure to a foreign substance (ink). These usually occur within the space of a few weeks and rarely require medical care, as the tattooists themselves can manage them. However, early reactions to tattoos can involve an infectious etiology [9]. Clinical data that are suggestive of infection include pain, a rise in local temperature, oozing, and ulceration (Figure 1). If these signs are present, the purulent discharge should be cultured, and patients should initiate empirical antibiotic treatment.

Allergic reactions to tattoo ink are late reactions. These are permanent lesions that do not respond to treatment with topical corticosteroids. The differential diagnosis should always
Table 1. Recommended History for a Skin Reaction in a Tattooed Area

Medical history
- History of contact allergies (especially to metallic salts or
textile dyes)
- Previous relevant dermatoses (eg, psoriasis, eczema,
urticaria, lichen planus, cutaneous lupus erythematous, and
pyoderma gangrenosum)
- Previous infections (eg, hepatitis, HIV)
- Systemic diseases (eg, sarcoidosis)
- Neoplastic diseases

Symptoms present

- How much time passed between completion of the tattoo and the appearance of the lesions?

- Is there pruritus, pain, or dysesthesia in the tattooed area?

- Does exposure to sunlight trigger or exacerbate the complaint?

- Is fever, arthralgia, lymph node involvement or another systemic manifestation present?

- What treatments have been used for this problem, and what were the results?

- Have any lesions appeared on previous tattoos?

Tattoo process

- When and where was the tattoo placed and who placed it (professional tattooist or amateur)?

- Were different colors used in the tattoo? To obtain the color(s), were the inks mixed?

- Do you have other tattoos? Is this the first time you were tattooed with this color ink?

- Can you get a sample of the problem ink and/or its safety data sheet?

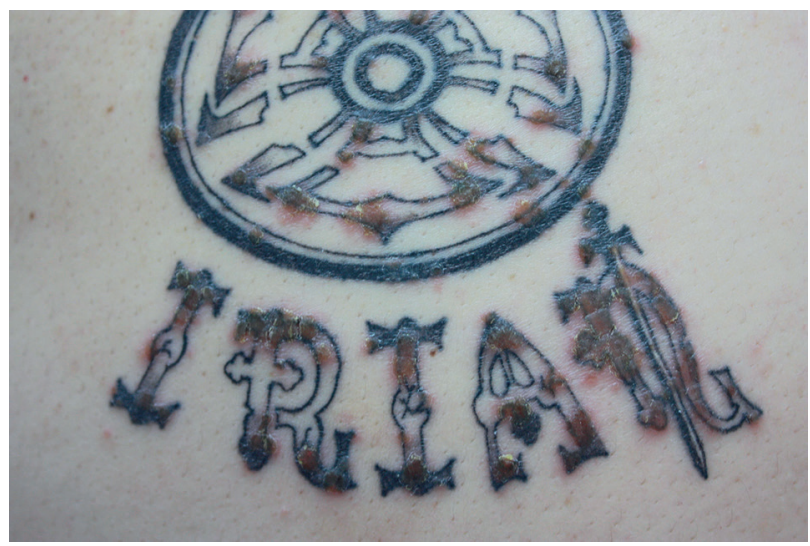

Figure 1. The presence of crusted honey-colored lesions distributed randomly in a recently tattooed area should lead us to suspect a bacterial infection.

consider other late reactions such as granulomatous foreign body reactions, other dermatoses, systemic disease (sarcoidosis) (Figure 2), microbial infections, pseudolymphomas, and 


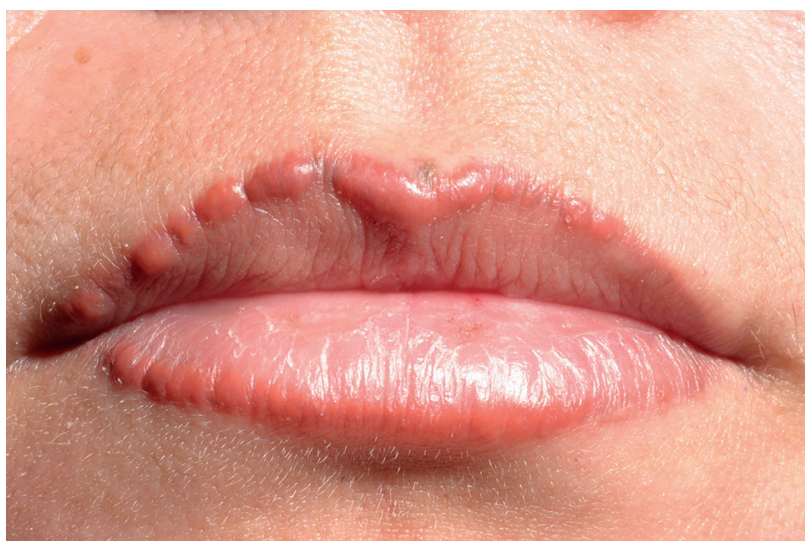

Figure 2. The appearance of sarcoidosis on previous cutaneous scars is a well-known phenomenon (scar sarcoidosis). It can also occur in tattooed areas and mimic a local reaction to the ink used in the tattoo.

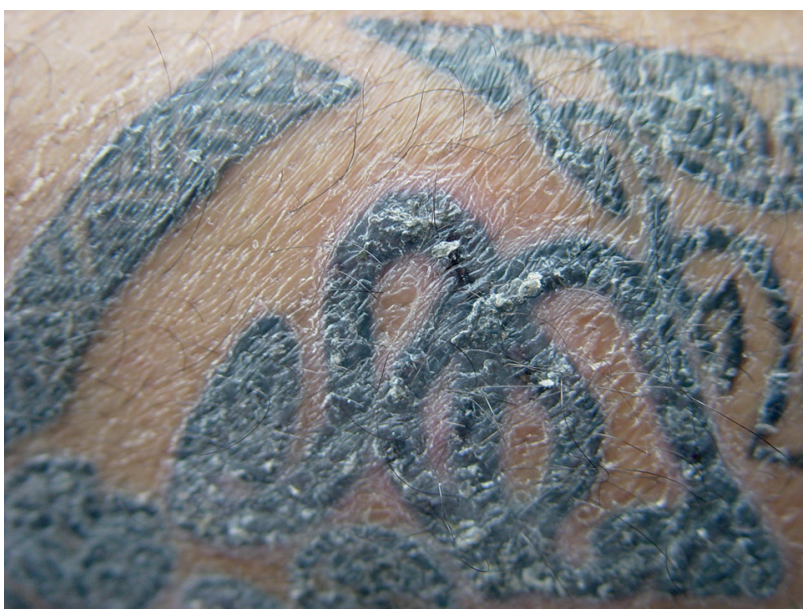

Figure 3. The late appearance of dry eczematous lesions with exclusive involvement of the tattooed area increases the suspicion of an allergic reaction.

neuropathic reactions. Establishing a diagnosis for an allergic reaction is not simple, as the skin lesions are nonspecific and the histological findings are not characteristic.

Serup et al $[9,10]$ developed a classification system for the clinical presentations associated with permanent tattoos to facilitate the practitioner's approach to affected patients. Rather than specific patterns, they are clinical forms that can orient clinicians toward various complications and include papulonodular, plaque-like, hyperkeratotic, ulceronecrotic, lymphopathic, neurosensory, and scar patterns. Advanced stages of any of these clinical patterns produce patches of scarring, with the disappearance of pigment or changes to its color.

The clinical patterns that are most frequently associated with allergic reactions are plaque-like, hyperkeratotic, and ulceronecrotic patterns. The plaque-like presentation is characterized by raised tissue in the areas tattooed with the problem color; the surface may be scaly (eczematous) (Figure 3) or smooth and shiny (lichenoid) (Figure 4) [11]. This pattern is uniform and homogeneous and is often seen in

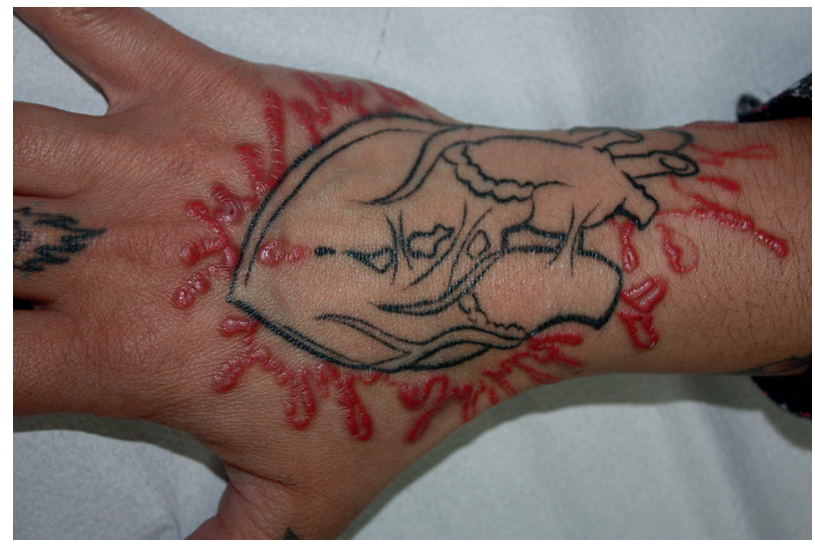

Figure 4. The appearance of flattened papular lesions (lichenoid pattern) that affect the entire tattooed surface of the same color is characteristic of allergic reactions to the inks used in tattoos.

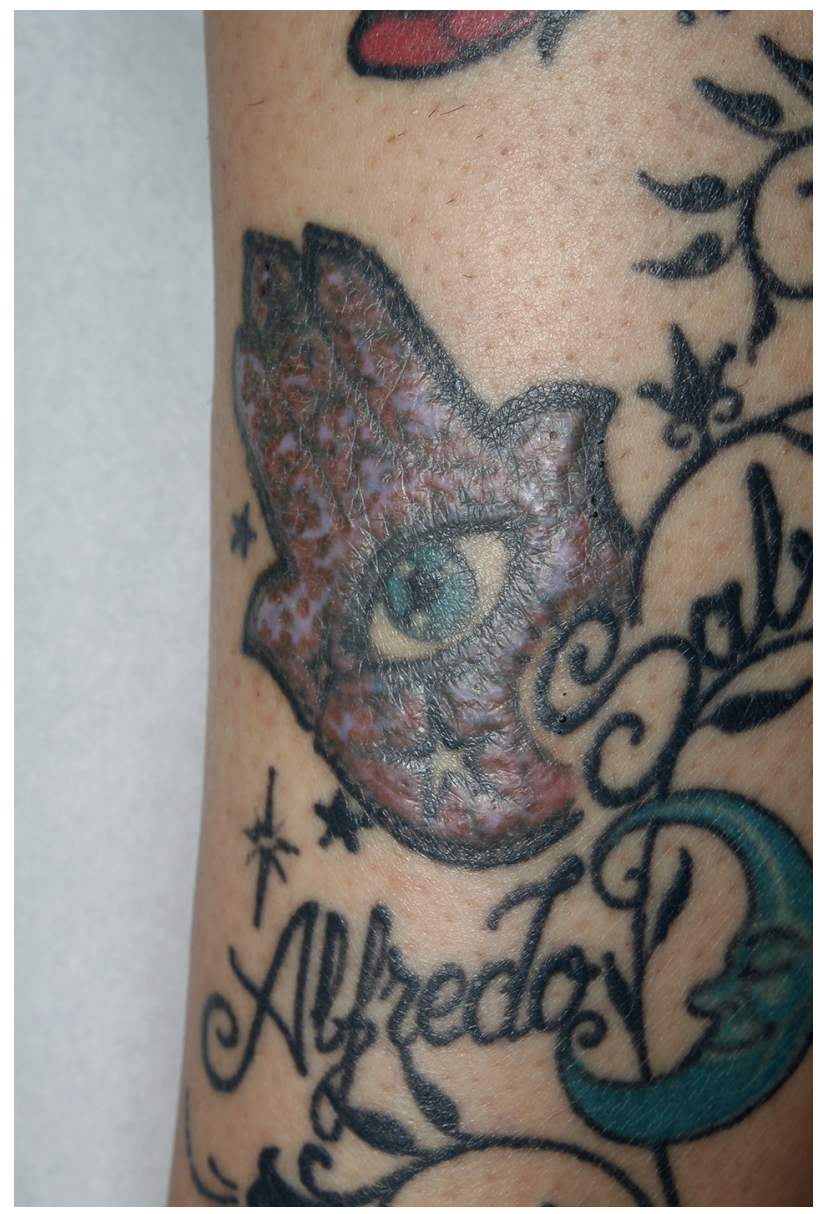

Figure 5. The presence of areas of scarring is a final stage to which most of these clinical patterns could progress.

tattoos with red ink, which is the most frequent cause of allergic reactions. Green and blue ink also tend to produce this pattern and may give way to color changes, ie, from blue to green or vice versa. The hyperkeratotic pattern entails thickening of the skin until it resembles sandpaper, with a flat surface that 
may ulcerate or necrotize. This pattern could be considered a variant of the plaque-like pattern, albeit with an excessive epidermal reaction caused by an inflammatory dermal cascade in response to the ink. This induces leakage of the pigment into the epidermis, thus activating the process of epidermal proliferation that gives rise to hyperkeratosis. The histological study reveals pseudoepitheliomatous hyperplasia. Finally, the last pattern that Serup et al $[9,10]$ link to an allergic reaction is the ulceronecrotic pattern. This manifestation arises from an aggressive inflammatory process resulting in ulceration of the areas tattooed with the culprit ink. This ulceration can affect the entire dermal layer and even the hypodermis. If the pigment spreads deep enough to reach the fascia of the muscle underneath or the regional lymphatic nodes, the inflammatory cascade can eventually provoke severe deep tissue necrosis or systemic autoimmune processes such as vasculitis or blistering

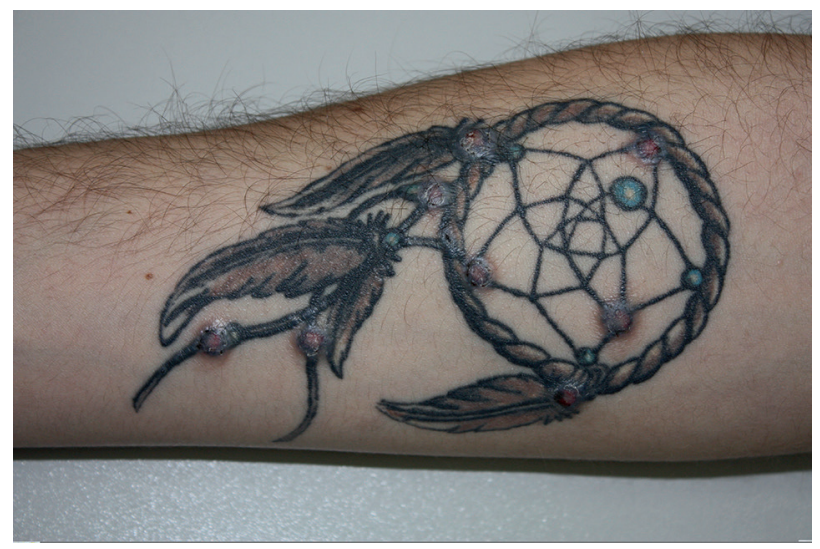

Figure 6. In this case, eczema-like lesions are observed in all red tattooed areas. The remaining colors do not present complications. Regardless of the clinical pattern, an essential finding to confirm a suspected allergic reaction is a uniform, monomorphic appearance throughout the area tattooed with the problem ink. Red ink is more commonly associated with complications than other colors.

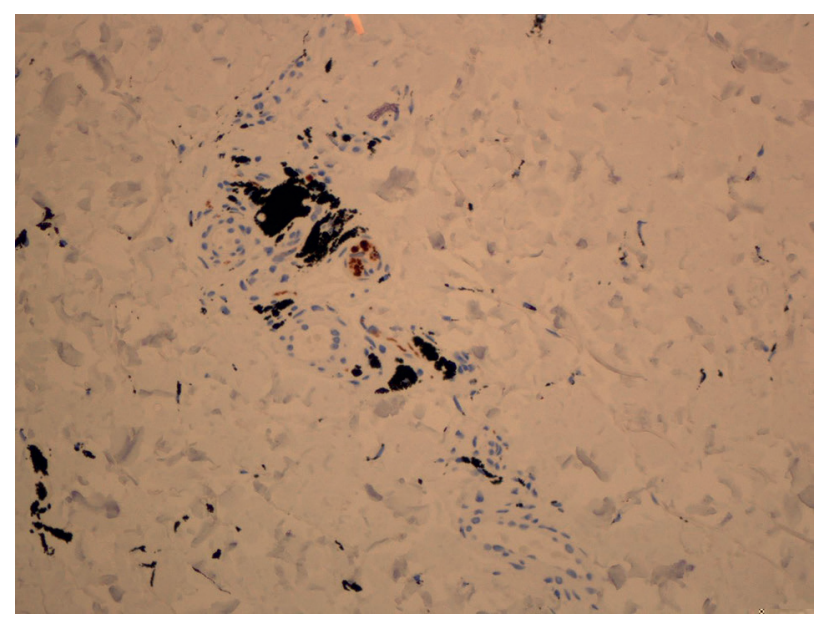

Figure 7. Deposition of the pigment of the ink around the sensory nerves of the dermis seems to be the origin of the discomfort that many patients experience in tattoos, apparently without lesions (neuropathic pattern). Allergy should not be suspected in these cases. diseases. All these patterns can evolve to a final stage in which there is loss of pigment, changes in color, and areas of scarring (Figure 5).

Regardless of the clinical pattern, an essential finding to confirm a suspected allergic reaction is a uniform, monomorphic appearance throughout the area tattooed with the problem ink (Figure 6). Clinicians need not suspect allergic reactions when there are localized spots of thickening (papulonodular pattern) in some areas but not others tattooed with a specific color of ink. Likewise, complaints of intense pruritus, pain, or dysesthesia on the tattoo-in the absence of skin lesions - do not indicate an allergic reaction. These
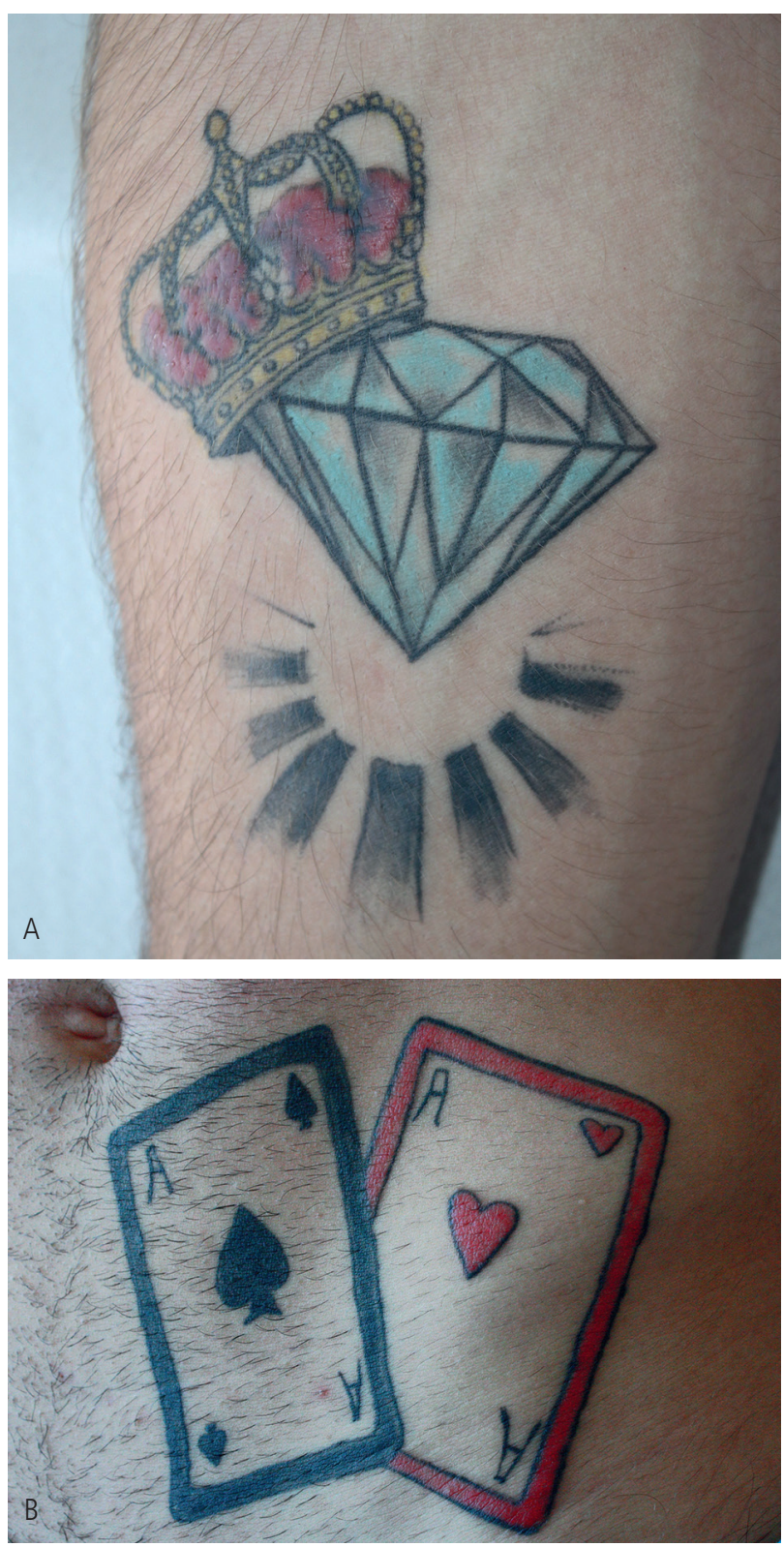

Figure 8. A, Eczematous plaque reaction in the red color of a tattoo placed a month and a half before the photo was taken. B, Similar simultaneous reaction in a red tattoo placed 2 years previously. This phenomenon is very suggestive of allergic reaction to tattoo ink. 
manifestations correspond to the neuropathic pattern, which is thought to be secondary to the stimulation of the C-fiber sensory nerves by degraded ink components (Figure 7) [12].

Another very useful clinical sign is the simultaneous appearance of similar reactions in both the problem tattoo and a previous tattoo of the same color. This finding is very suggestive of an allergic reaction (Figure 8). Table 2 shows all of the clinical signs suggesting an allergic reaction to tattoo inks [11].

Allergic reactions tend to present locally, but some authors have described cases of generalized rashes or eczemas, especially in previously sensitized patients. These reactions appear early (within 1-2 days of being tattooed) and tend to resolve without treatment after a few weeks or months [8]. Other authors have described cases of generalized reactions upon attempting to eliminate the pigment with laser treatment [13], and cases of photoallergic reactions in tattoos with yellow ink containing cadmium [1].

A final consideration is the fact that tattooing is an inherently aggressive process, and this can trigger the appearance of certain dermatoses on the tattooed skin (isomorphic phenomenon of Koebner), including psoriasis, lichen planus, cutaneous lupus erythematous, and pyoderma gangrenosum (Figure 9). Recognizing these situations is straightforward when a patient has a prior diagnosis, although they may also present as new-onset lesions.

Table 2. Clinical Signs of Allergic Contact Dermatitis to Tattoo Ink

LATE reaction (weeks, months, or years after being tattooed) PERSISTENT reaction

HOMOGENEOUS involvement of ALL AREAS where the ink of the same COLOR was injected

Clinical pattern: PLAQUE-LIKE, LICHENOID,

HYPERKERATOTIC, or ULCERO-NECROTIC

DISTANT REACTION in OTHER PREVIOUS TATTOOS of the SAME COLOR

POOR RESPONSE to topical corticosteroids

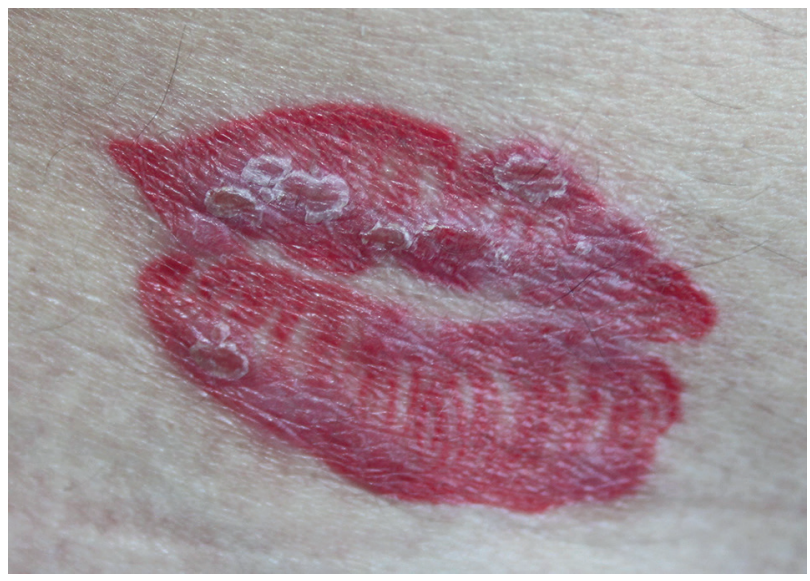

Figure 9. Psoriasis presents as an isomorphic phenomenon and may appear on previously injured skin such as scars, surgical wounds, or tattoos.

\section{Histology Findings}

Skin biopsy is a crucial part of the study of any late or persistent reaction in the tattooed area. Histopathology of the samples can rule out serious entities such as cutaneous infections by atypical microorganisms, systemic diseases,

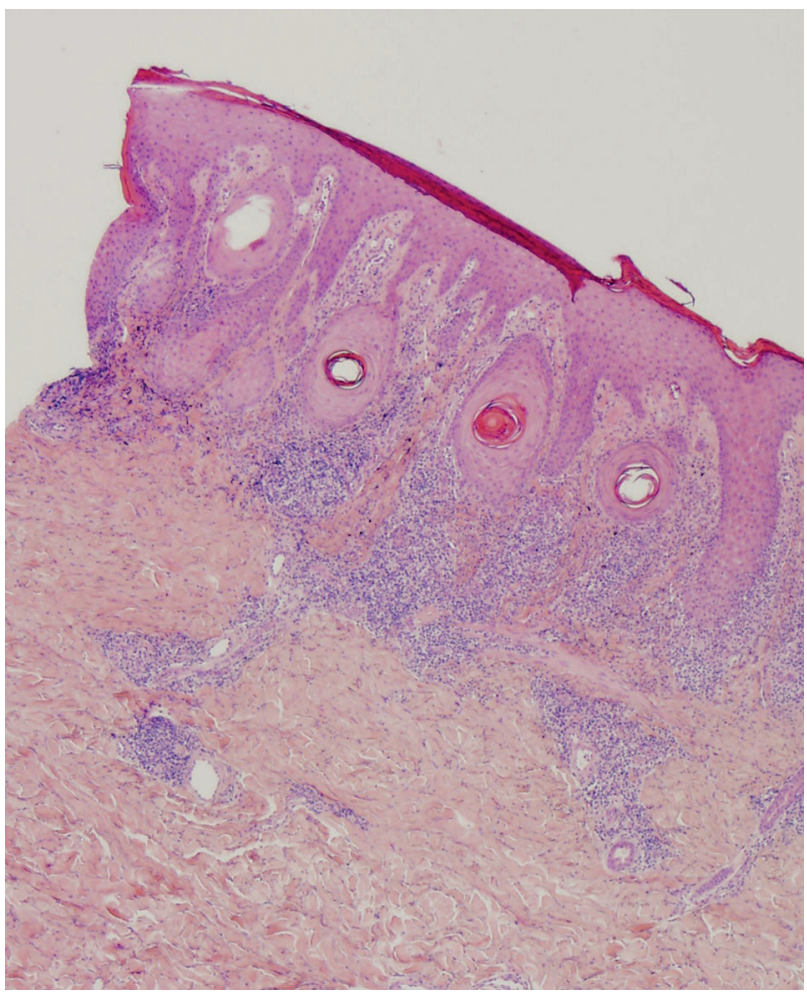

Figure 10. The presence of interface dermatitis with predominantly lymphocytic band-like inflammatory infiltrate in the dermoepidermal junction and in the superficial dermis, whether associated or not with spongiotic dermatitis, is a frequent histological finding in allergic reactions to tattoo ink.

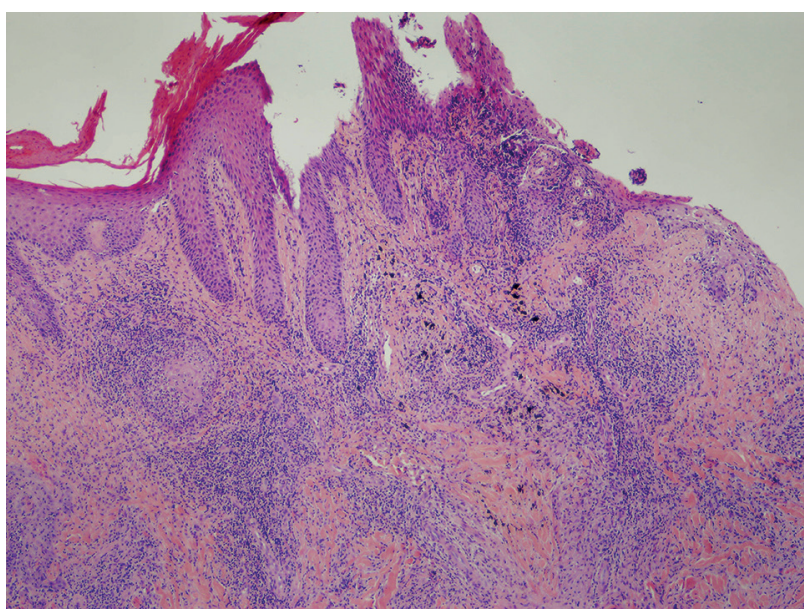

Figure 11. Pseudoepitheliomatous hyperplasia with transepidermal pigment elimination is a histological finding suggestive of allergic reaction to tattoo ink. 
or lymphomatous tumor infiltrations [14]. It is important to remember that since allergic reactions have no pathognomonic histological findings, clinicians should not expect to see a histological pattern of spongiotic dermatitis, as with allergic contact eczema, because the allergen is directly inoculated into the dermis. The indication that is most suggestive of allergy is interface dermatitis, with predominantly lymphocytic inflammatory infiltrate, with or without eosinophils. This bandlike inflammatory infiltrate occupies the basal layer of the epidermis and the papillary dermis (Figure 10). It is made up of mononuclear cells and macrophages loaded with intracellular pigment. Dermal fibrosis is apparent to some extent, especially in advanced stages. The immunohistochemical study shows a predominance of $\mathrm{T}$ lymphocytes that are $\mathrm{CD}^{+}$and also $\mathrm{CD} 4^{+}$ or $\mathrm{CD}^{+}$; both populations are frequent in allergic inflammatory infiltrates $[15,16]$.

In many cases, attempted transepidermal elimination of the pigment and even pseudoepitheliomatous hyperplasia can be observed (Figure 11). Given that this has been described as a pattern of response to various infectious processes, stains are recommended as a means of ruling out the presence of fungi, bacteria, and microbes [1].

More rarely, allergic reactions to tattoos can present with histological patterns that are granulomatous or pseudolymphomatous (both B- and T-cell types). Thus, biopsies showing one of these patterns are insufficient to exclude the diagnosis of an allergic reaction. In the presence of a granulomatous pattern, clinicians should rule out systemic granulomatous diseases and microbial or fungal infections. In the case of pseudolymphoma, we must demonstrate that the inflammatory infiltrate is polyclonal by means of molecular biological studies $[1,7,11,17]$.

\section{Patch Testing}

Patch testing is used for the study of allergic contact dermatitis, which is caused by haptens that come into contact with the epidermis and are capable of eliciting a delayed immune response mediated by lymphocytes. It is not clear whether this test is useful for diagnosing delayed hypersensitivity reactions provoked by antigens that are inoculated directly into the dermis. However, it is true that many cases of (noneczematous) lichenoid reactions to metals have been described, as well as drug eruptions with positive patch tests, in which the allergen has not penetrated through the epidermis but was instead administered systemically [18]. Theoretically, intradermal tests would be the most specific for the study of these reactions; however, they are not risk-free. Permanent pigment may remain in the dermis following the tests, or a chronic reaction may be triggered. Thus, performing these tests is not considered appropriate or ethical [19,20].

Patch testing in the presence of a suspected tattoo allergy has often been undertaken without knowledge of the ingredients in the ink used. Indeed, in many countries, ink manufacture and composition are still unregulated, with no labelling obligations. This is not the case in Spain, where the Spanish Agency of Medicines and Medical Devices approves tattoo inks for marketing and mandates labelling of their ingredients [21]. Thus, faced with a suspected tattoo allergy,

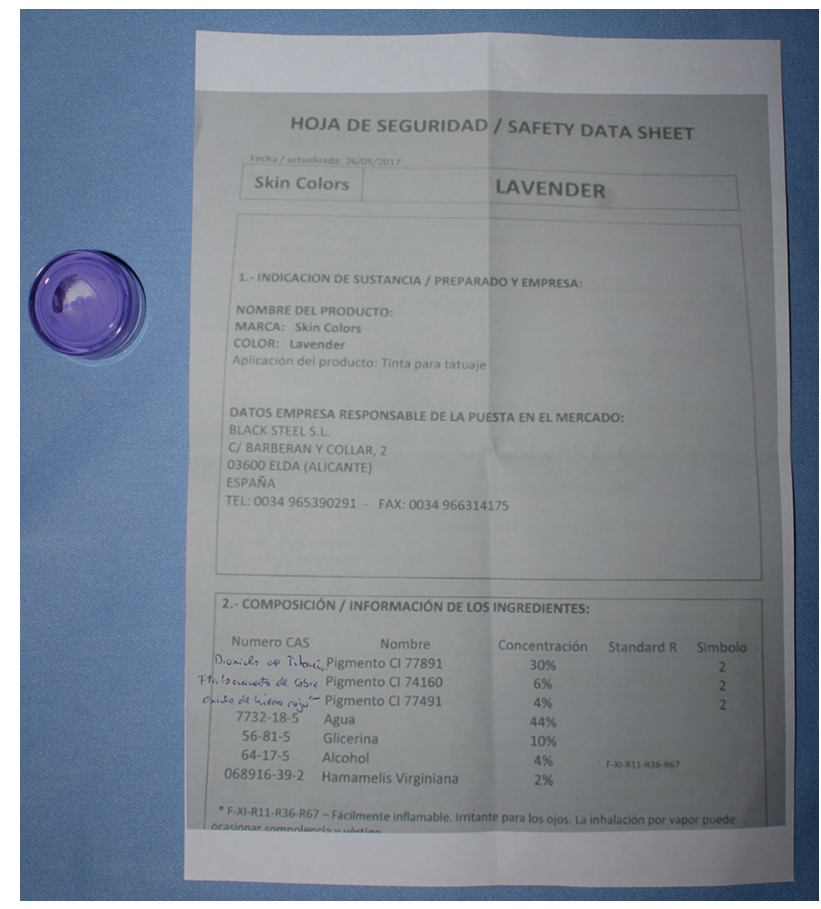

Figure 12. Prior to performing patch testing, it is advisable to obtain the problem ink and its safety data sheet.

clinicians can and should request the safety data sheets and batch numbers for the products used by the tattooist (Figure 12). In clinical practice, these are not always easy to obtain; securing this information depends on the collaboration of both the patient and the tattooist. The patient often fails to obtain the ink, or the tattooist might provide an unlabeled sample that may or may not be the one used for the tattoo. The patient may even provide safety sheets detailing identical compositions for the entire array of pigments sold under a particular brand name.

It is not clear which allergens should be studied through patch tests for suspected tattoo allergy; patch tests are mainly performed for potential allergens identified from inks on other occasions, albeit with no certainty that they are present in the problem ink [22]. We usually apply the Spanish baseline series, sometimes adding a specific series of metals and/or textile dyes. If possible, we also patch test the problem ink and the potential allergens listed on the safety sheet (including auxiliary ingredients and excipients) [18].

The metals series is used because inks may contain pigments from inorganic metal salts; the most frequent of these are titanium (white ink) along with barium and iron (red), although other salts that may be present include those from mercury (red), cadmium (yellow, red), zinc (white), nickel and chrome (green), cobalt (blue), aluminum and manganese (purple), and gold $[1,11]$. The metal that has been most frequently identified as an allergen is mercury, which was a component of red ink. Today, metallic salt components, which used to be very common, are giving way to organic molecules such as azo pigments, which could now be present in up to $60 \%$ of color inks [23]. The textile dyes series includes a number of azo pigments, which is why this series tends to be used in patients with cutaneous reactions to tattoos, even 
though we are not sure that the series contains the azo colors used in the tattoo [24].

Few studies reflect the utility of patch testing in these situations. Most published articles correspond to isolated clinical cases with positive results and an adequate clinical correlation. Serup et al [25] undertook patch testing in 90 patients with chronic reactions to tattoos, although the results were discouraging. The baseline series showed positive findings for metal allergens that the authors determined were not relevant (Figure 13), and the specific series for dyes and the problem ink showed positive results for only $5 \%$ and $8 \%$ of the cases, respectively (Figure 14) [25].

This lack of sensitivity and specificity to patch testing in the detection of allergic reactions to tattoo inks has prompted several hypotheses. Some authors propose that the scant penetration of the test ink into the skin is the reason for the negative results. More recently, investigators speculated that the real allergen was not present in its native form in the ink itself, but rather was generated in the dermis from pigments or other ink components through a haptenization process that could take weeks, months, or even years. The culprit allergen could even be a metabolite. The concentration of azo pigments in the skin has been shown to decrease over time, as the
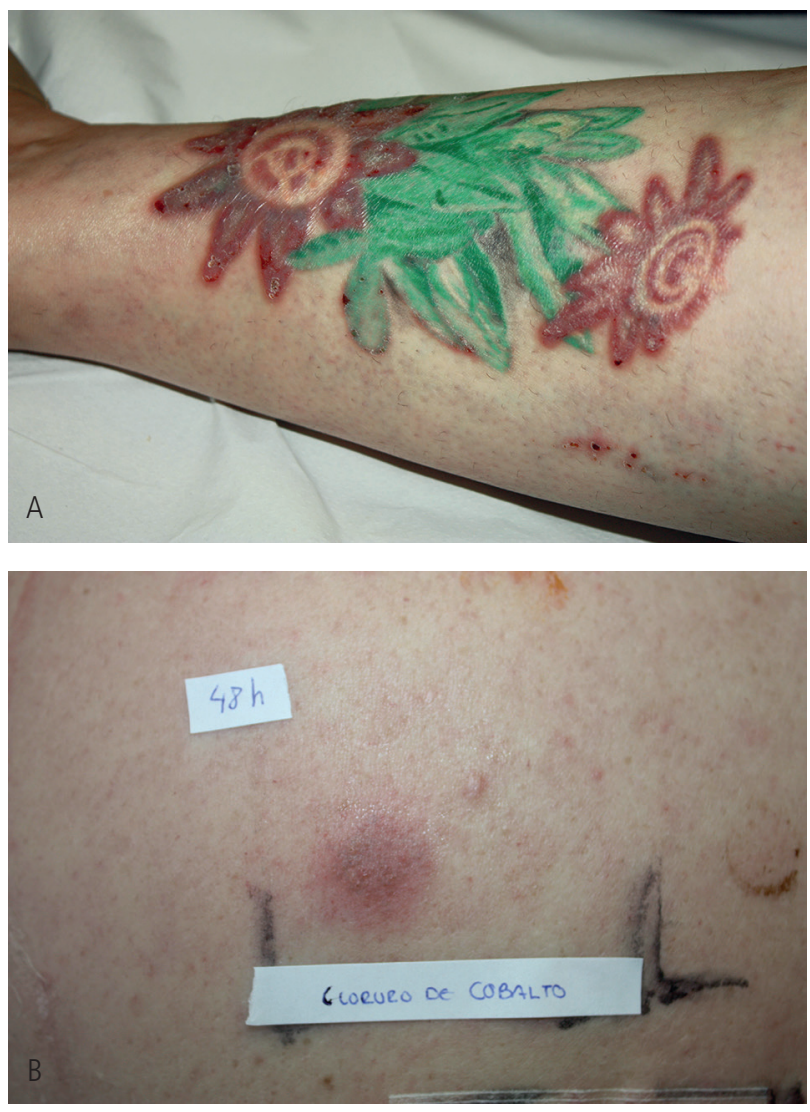

Figure 13. A, Patient with eczematous skin reaction to "apparently" red tattoo ink. B, Results of patch testing with positivity to cobalt chloride. The patient told us that she had previously been tattooed with a blue ink, but that she was retattooed with a red ink in order to improve the appearance of the tattoo. No relevance could be established, given that it was unknown whether the blue ink contained cobalt chloride.

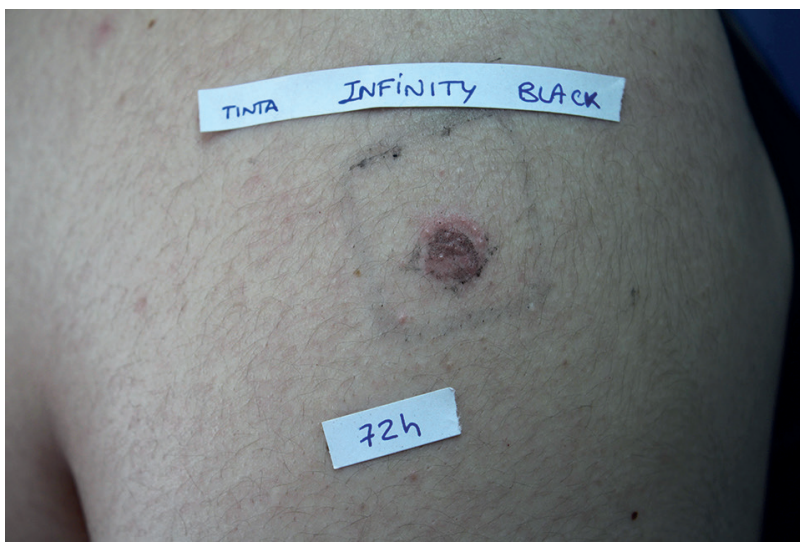

Figure 14. Patch testing with the problem ink (as is) is advisable, although it is generally negative. In this case, it was positive, and we discovered that the patient was allergic to shellac, which is used to bind the pigment particles in black inks.

pigments are transported in part by the lymphatic system and in part degraded in the dermis into products such as aminonaphthol-AS and naphthol-AS. These metabolic products could potentially be the ones responsible for the allergic reactions [26]. However, they have been tested in 3 patients, with negative results [23]. Some researchers have postulated that sunlight could induce a process of photochemical cleavage of the tattoo pigments, particularly the azo pigments, which would enable chemical substances to act as haptens [27,28]. This observation could explain numerous phenomena of solar intolerance that have been described in tattooed areas and opens up new possibilities in how to approach these patients, whether through photopatch testing or patch testing with substances processed with ultraviolet light. Two studies have explored these additional tests, both with negative results [29,30]. A further consideration is that tattoo inks also contain other auxiliary ingredients and excipients, such as propylene glycol and shellac, which could also be the culprit allergens and should be considered in patch testing [31].

\section{Diagnostic Algorithm}

Based on our experience, we propose an algorithm for managing patients with reactions to a tattoo (Figure 15). Our approach depends initially on whether the lesion presents early or late [6].

If an early reaction in the area of the tattoo shows clinical signs of infection, it is essential to perform a culture of the purulent discharge as well as a biopsy for a histological and microbiological study (bacterial and microbial culture). Empirical antibiotics can also be started. If a sample of the ink used is available, it would be desirable to perform a culture on it to rule out contamination. For early reactions without clinical signs of infection, we recommend initiating treatment with topical corticosteroids. If the reaction is refractory to treatment, a biopsy is warranted.

In cases of late reactions, we recommend undertaking a skin biopsy to rule out infection, systemic granulomatous diseases, and lymphatic neoplasms. If the biopsy shows a 


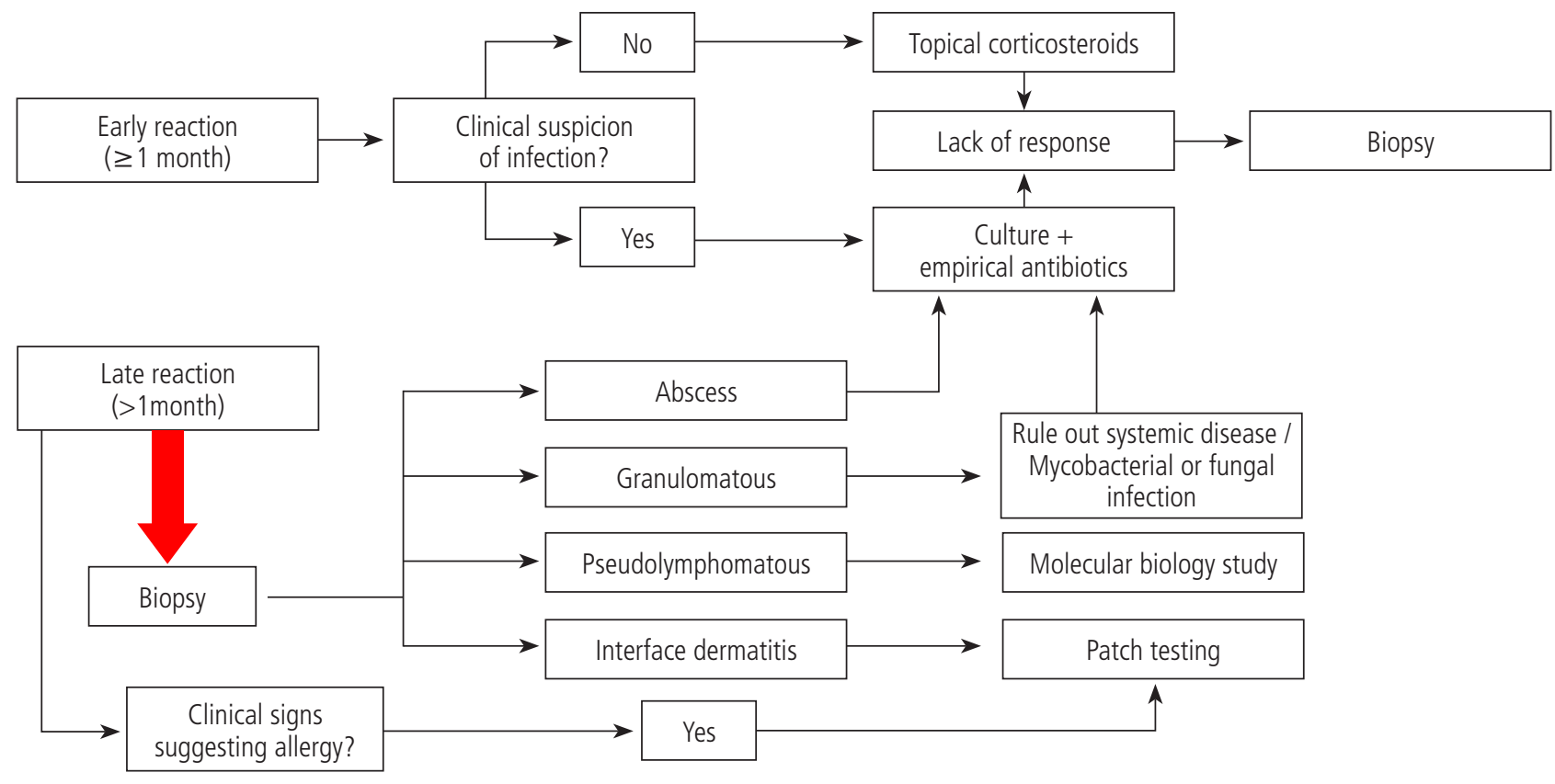

Figure 15. Diagnostic algorithm for managing patients with cutaneous reactions to a tattoo.

lichenoid or spongiotic pattern and the patient shows clinical signs that are compatible with an allergic reaction, we advise performing patch tests with at least the Spanish baseline series, and if possible, also with the problem ink and all of the ingredients listed on the safety data sheet. If the biopsy reveals an abscess, a sample should be sent for culture, whereas a granulomatous pattern would require us to rule out systemic disease and send a sample for fungal and microbial culture. In patients with a histological pattern of B- or T-cell pseudolymphoma, a molecular study is advised.

\section{Conclusions}

Diagnosis of allergic reactions to ink in permanent tattoos is very challenging. While clinical and histological data can help clinicians recognize the reactions, these indications are not specific. We consider that biopsy is essential in late reactions, and while we also recommend performing patch testing, this approach currently has a low yield. We hope that more information on the composition of tattoo inks will be available in the future, so that clinicians can choose allergens for patch tests more accurately and thus identify the likely cause of allergic reactions to tattoos.

\section{Funding}

The authors declare that no funding was received for the present study.

\section{Conflicts of Interest}

The authors declare that they have no conflicts of interest.

\section{References}

1. Mataix J, Silvestre JF. Reacciones cutáneas adversas por tatuajes y piercings. Actas Dermosifiliogr. 2009;100:643-56.

2. Kluger N. Cutaneous complications related to tattoos: 31 cases from Finland. Dermatology. 2017;233:100-9.

3. Hogsber T, Hutton K, Serup J. High prevalence of minor symptoms in tattoos among a young population tattooed with carbon black and organic pigments. J Eur Acad Dermatol Venereol. 2013;27:846-52.

4. Kluger N. Self-reported tattoo reactions in a cohort of 448 French tattooists. Int J Dermatol. 2016;55:764-8.

5. Brady BG, Gold H, Leger EA, Leger MC. Self-reported adverse tattoo reactions: a New York City Central Park study. Contact Dermatitis. 2015;73:91-9.

6. González-Villanueva I, Silvestre Salvador JF. Diagnostic Tools to Use When We Suspect an Allergic Reaction to a Tattoo: A Proposal Based on Cases at Our Hospital. Actas Dermosifiliogr. 2018:109:162-72

7. Serup J. Diagnostic tools for doctors' evaluation of tattoo complications. Curr Probl Dermatol 2017;52:42-57.

8. González I, Silvestre JF. Claves clínicas ante una sospecha de reacción alérgica a un tatuaje. Piel. 2018;33:575-80.

9. Serup J, Carlsen KH, Sepehri M. Tattoo complaints and complications: diagnosis and clinical spectrum. Curr Probl Dermatol. 2015;48:48-60.

10. Serup J. How to diagnose and classify tattoo complications in the clinic: a system of distinctive patterns. Curr Probl Dermatol. 2017; 52:58-73.

11. González I, Silvestre JF. Reacciones alérgicas frente a las tintas de los tatuajes. In: Muñoz D, Ed. Tratado sobre los tatuajes. 
Claves para su eliminación con láser. Buenos Aires: Ediciones Journal, 2017; p. 69-76.

12. Morte PD, Magee M. Hyperalgesia after volar wrist tattoo: a case of complex regional pain syndrome? J Clin Neuromuscul. 2011;12:118-21.

13. Zemtsov A, Wilson L. CO2 laser treatment causes local tattoo allergic reaction to become generalized. Acta Derm Venereol. 1997;77:497.

14. Simunovic C, Shinohara MM. Complications of decorative tattoos: Recognition and management. Am J Clin Dermatol. 2014;15:525-36.

15. Romani N, Clausen B, Stoitzner P. Langerhans cells and more: langerin-expressing dendritic cell subsets in the skin. Immunol Rev. 2010;234:120-41.

16. Kimber I, Dearman RJ, Basketter DA. Dendritic cells and the assessment in vitro of skin sensitizing potential. Cutan Ocul Toxicol. 2013;32:54-9.

17. Høgsberg T, Thomsen BM, Serup J. Histopathology and immune histochemistry of red tattoo reactions. Skin Res Technol. 2015;21:449-58.

18. Silvestre JF, González I. Utilidad de las pruebas epicutáneas en el estudio de las reacciones cutáneas a tatuajes. Piel. 2018:33:549-51.

19. McFadden N, Lyberg T, Hensten-Pettersen A. Aluminuminduced granulomas in a tattoo. J Am Acad Dermatol. 1989;20:903-8

20. Gutermuth J, Hein R, Fend F, Ring J, Jakob T. Cutaneous pseudolymphoma arising after tattoo placement. J Eur Acad Dermatol Venereol. 2007;21:566-7.

21. Michel R. Manufacturing of tattoo ink products today and in future: Europe. Curr Probl Dermatol. 2015;48:103-11.

22. Serup J, Kluger N, Bäumler W. Tattoo inks: legislation, pigments, metals and chemical analysis. Curr Probl Dermatol. 2015:48:152-7.

23. Gaudron S, Ferrier-Le Bouëdec MC, Franck F, D'Incan M. Azo pigments and quinacridones induce delayed hypersensitivity in red tattoos. Contact Dermatitis. 2015;72:97-105.
24. González-Villanueva I, Álvarez-Chinchilla P, Silvestre JF. Allergic reaction to 3 tattoo inks containing Pigment Yellow 65. Contact Dermatitis. 2018;79:107-8

25. Serup J, Hutton Carlsen K. Patch test study of 90 patients with tattoo reactions: negative outcome of allergy patch test to baseline batteries and culprit inks suggests allergens are generated in the skin through haptenization. Contact Dermatitis. 2014;71:255-63.

26. BäumlerW. Absorption, distribution, metabolism and excretion of tattoo colorants and ingredients in mouse and man: the known and the unknown. Curr Probl Dermatol. 2015;48:17684.

27. Engel $E$, Spannberger $A$, Vasold $R$, König Landthaler $M$, Bäumler W. Photochemical cleavage of a tattoo pigment by UVB radiation or natural sunlight. J Dtsch Dermatol Ges. 2007:5:583-9.

28. Hutton Carlsen K, Serup J. Photosensitivity and photodynamic events in black, red and blue tattoos are common: a 'Beach study'. J Eur Acad Dermatol Venereol. 2014;28:231-7.

29. Tresukosol P, Ophaswongse S, Kullavanijaya P. Cutaneous reaction to cosmetic lip tattooing. Contact Dermatitis. 1997;36:176-7.

30. Steinbrecher I, Hemmer W, Jarisch R. Adverse reaction to the azo dye Pigment Red 170 in a tattoo. J Dtsch Dermatol Ges. 2004;(2):1007-8.

31. González-Villanueva I, Hispan P, Silvestre JF. Allergic contact dermatitis caused by a black tattoo ink in a patient allergic to shellac. Contact Dermatitis. 2016;75:247.

\section{Juan Francisco Silvestre Salvador}

Pintor Baeza 12

03010 Alicante, Spain

E-mail: silvestre_jfr@gva.es 